\title{
Bacteriology of blood stream infections in patients on hemodialysis and antibiotics susceptibility pattern
}

\begin{abstract}
Background: Bacteriological blood stream infection is the most common cause of mortality in hemodialytic patients as they are inmmunocompromised. In such patients blood stream infections is mainly due to gram positive cocci. Patients under the course of haemodialysis are particularly vulnerable if it is due to Staphylococcus aureus that accounts more than $8 \%$ mortality rate.
\end{abstract}

\begin{abstract}
Methods: A cross-sectional study was carried out at National Kidney Center of Nepal for duration of six month. Blood sample collected from the patients undergoing hemodialysis course was inoculated in brain heart infusion biphasic media in blood culture bottle and incubated aerobically at $37^{\circ} \mathrm{C}$ for $18-24$ hours (up to 1 week if necessary). It was than subcultured on blood agar plate where colony morphology and haemolytic reactions was observed. A set of conventional biochemical tests was performed to identify the isolated organisms. Antimicrobial susceptibility test was carried out by modified Kirby Bauer technique. All obtained data were recorded and analyzed using SPSS software.
\end{abstract}

Volume 2 Issue 5 - 2015

\author{
Shrestha Prasansah,' Pokharel Nabaraj,' Pant \\ Anil $\mathrm{Dev}^{2}$ \\ 'National College, Nepal \\ ${ }^{2}$ National Kidney Center, Nepal
}

Correspondence: Prasansah Shrestha, Department of Microbiology, National College, Khusibu, Kathmandu, Nepal, Tel +977941485068, Email praisehonour@gmail.com

Received: July 27, 2015 | Published: October 08, 2015

Results: Gram-positive bacteria were isolated with the highest frequency $(88.5 \%)$ Among them Staphylococcus aureus (69.6\%) was found predominant. The prevalence of gram negative bacteria was just $11.5 \%$ they were Escherichia coli, Klebsiella spp. and Pseudomonas aeruginosa. During antimicrobial susceptibility testing, most of the Staphylococcus aureus were found resistant to amoxicillin and sensitive to cephoxitin. Minimum inhibitory concentration of this antibiotic against Staphylococcus aureus was determined to be 64 and $128 \mathrm{mg} / \mathrm{L}$. All coagulase negative staphylococci were found resistant to $\beta$-lactam antibiotics and most of them were sensitive to ciprofloxacin. Out of total 16 Staphylococcus aureus isolates $37.5 \%$ were found to be methicillin resistant.

Conclusion: Among the gram positive isolates Staphylococcus aureus was found to be predominant organisms in hemodialytic patients. And most of these $S$. aureus were found resistant to amoxicillin and sensitive to cephoxitin. Out of total $16 \mathrm{~S}$. aureus isolates $37.5 \%$ were found to be methicillin resistant. Amoxicillin was found to be least effective drug and ciprofloxacin was found most effective drug for most gram positive bacteria from HD patient.

Keywords: AST, MRSA, CoNS, HD

\section{Background}

Patients requiring hemodialysis are at high risk for blood stream infection (BSI) mainly due to Gram- positive bacteria.. Within gram positive bacteria Staphylococcus aureus (S. aureus) and coagulase negative staphylococci (CoNS) are most common. ${ }^{1}$ BIS due to methicillin resistant $S$. aureus (MRSA) has been found to have a higher attributable mortality than methicillin susceptible $S$. aureus (MSSA). ${ }^{2}$ Although the incidence of MRSA is emerging threat, there is no study of BSI in persons under the course of HD in Nepal. Besides, the etiology of BSI is poorly characterized in this country, mainly due to limited laboratory resources, a poor recording system and an inadequate number of trained personnel. This study was thus designed to investigate the antibiotic resistance patterns of bacterial isolates during blood culture from HD patients.

\section{Methods}

\section{Ethical considerations}

This study was conducted with the approval of the committee of National College and National Kidney Center and declaration of
Helsinki was strictly followed.

\section{Design, period and location of the study}

A cross-sectional study was conducted at National Kidney Center, Kathmandu, Nepal from June 2013 to December 2013. All the patients who had attended this kidney center for hemodialysis and agreed to provide the blood sample after informed consent were included in this research. However, HD patients with HIV or Hepatitis C were not included.

After obtaining informed consent, $5 \mathrm{ml}$ of blood sample was collected aseptically and inoculated in $45 \mathrm{ml}$ sterile brain heart infusion (BHI) biphasic media. The BHI biphasic media culture bottles were labeled and incubated at $37^{\circ} \mathrm{C}$. Incubation was continued for up to 7 days unless the visible growth was observed. After getting a visible sign of microbial growth, it was subcultured on blood agar and MacConkey agar plate medium for isolation and identification of the organisms. Isolates were identified using standard microbiological techniques as describe by Forbes et al., ${ }^{3}$ using cultural, morphological and biochemical tests. 
The antibiotic susceptibility pattern of all the isolates was determined by modified Kirby Bauer disc diffusion method. The antibiotics were selected and the results were interpreted according to CLSI 2012 guidelines. Strains of $S$. aureus resistant to cefoxitin $(\mathrm{CX} / 30 \mathrm{mcg})$ and oxacillin $(\mathrm{OX} / 1 \mathrm{mcg})$ were considered as MRSA strain.

The statistical analysis was formed using SPSS version 17. The Chi-square test was used to calculate probabilities and determine significance. $\mathrm{P}$ value $<0.05$ was considered to be statistically significant.

\section{Results}

Out of 100 blood samples subjected for culture $26 \%$ showed culture positive result. Among these positive cases, $43.3 \%$ were male. The prevalence of BSI was found to be highest in the age group 45-59 years. And there is no significant association between the age group of patients and culture positive result (Table 1).
Table I Blood culture pattern of patients, according to age

\begin{tabular}{|c|c|c|c|c|c|c|}
\hline \multicolumn{5}{|c|}{ Age of patients } & \multirow{2}{*}{ Total } & \multirow{2}{*}{$\begin{array}{l}P \\
\text { value }\end{array}$} \\
\hline Status & $15-29$ & $30-44$ & $45-59$ & $\geq 60$ & & \\
\hline $\begin{array}{l}\text { Culture } \\
\text { Positive (\%) }\end{array}$ & 26.9 & 19.2 & 38.5 & 15.4 & 100 & \\
\hline $\begin{array}{l}\text { Culture } \\
\text { Negative (\%) }\end{array}$ & 21.6 & 28.4 & 20.3 & 29.7 & 100 & 0.177 \\
\hline Total & 48.5 & 47.6 & 58.8 & 45.1 & 200 & \\
\hline
\end{tabular}

Among the total $88.5 \%$ Gram positive isolates, $62.5 \%$ S. aureus isolates were found resistant to amoxicillin and cotrimoxazole. On the other hand, the most effective drug for the isolates was found to be cephoxitin (62.5\%). But all CoNS isolates $(30.5 \%)$ were found to be resistant to amoxicillin and oxacillin. Among Gram negative isolates $66.7 \%$ were sensitive to ofloxacin but all were resistant to cotrimoxazole (Table 2).

Table 2 Antibiotics susceptibility pattern of Gram positive and Gram negative organisms in percentage

\begin{tabular}{|c|c|c|c|c|c|c|c|c|c|c|}
\hline Organisms & AMX & C & CIP & $\mathbf{C N}$ & СОT & CTR & CTX & GEN & OF & Ox \\
\hline \multicolumn{11}{|l|}{ For Sensitive: } \\
\hline Gram positive S. aureus $(n=16)$ & 25 & 31.2 & 50 & 62.5 & 37.5 & 37.5 & 56.3 & 43.7 & - & 12.5 \\
\hline CoNS $(n=7)$ & 0 & 42.8 & 71.4 & 42.8 & 28.6 & 28.6 & 57.1 & 57.1 & - & 0 \\
\hline Gram negative & 33.33 & 0 & 33.33 & - & 0 & 33.33 & 0 & 33.33 & 66.66 & - \\
\hline \multicolumn{11}{|l|}{ For Resistance: } \\
\hline Gram positive S. aureus $(n=16)$ & 62.5 & 31.2 & 31.2 & 37.5 & 62.5 & 43.7 & 37.5 & 50 & - & 81.2 \\
\hline CoNS $(n=7)$ & 100 & 28.6 & 0 & 42.8 & 57.1 & 71.4 & 28.6 & 14.3 & - & 100 \\
\hline Gram negative & 33.33 & 66.66 & 0 & - & 100 & 33.33 & 66.66 & 0 & 0 & - \\
\hline
\end{tabular}

AMX, amoxicillin; C, chloramphenicol; CIP, ciprofloxacin; CN, Cephoxitin; COT, cotrimoxazole; CTR, ceftriaxone; GEN, gentamycin; OF, ofloxacin; OX, oxacillin

\section{Discussion}

In this study from $26 \%$ sample processed and these samples were considered culture positive cases. No organism were detected from $74 \%$ blood samples processed until 1 week of incubation at $37^{\circ} \mathrm{C}$ and these cases were considered as culture negative.

Difference in the prevalence of BSI in HD patients of different age group was found statistically insignificant.

Gram positive bacteria were found highly prevalent than gram negative bacteria. It can also be revealed that more than half $(61.5 \%)$ of the bacteriological blood stream infection episodes is due to S. aureus. Similar finding was also reported by Fysaraki et al., ${ }^{4}$ in hemodialytic patients. According to Jaber $^{5}$ and Taylor et al., ${ }^{6}$ the majority cases of the BSIs are due to gram positive microorganisms especially skin-derived $S$. aureus and $S$. epidermidis. Similar finding was also reported by Chaudhry et al., ${ }^{7}$ and Lee et al. ${ }^{8}$ According to Thiene, ${ }^{9}$ the gram positive organisms are comparatively resistant to bactericidal activity of serum than gram negative and this could be the reason behind gram positive being the most common etiological agent of bacterial BSIs.
Diekema et al., ${ }^{10}$ reported that, gram positive bacteria specially $S$. aureus cause majority of both nosocomial and community acquired BSIs however, the rank of pathogen is different for community and nosocomial infections.

Gram negative isolates though less frequently isolated were found more sensitive to ofloxacin and all of them were found resistant to cotrimoxazole. The gram positive isolates were tested for different groups of antibiotics generally used for the treatment purpose. Cephoxitin, ciprofloxacin and gentamycin were found to be most effective for gram positive isolates and oxacillin was found to least effective antibiotic for those gram positive organisms. Similar finding had been reported by Manandhar. ${ }^{11}$ Strains of $S$. aureus have developed resistance to antibiotics including penicillin, cephalosporins, methicillin, aminoglycosides and quinolones. $S$. aureus abrogates the effects of penicillin by producing $\beta$-lactamase and MRS strains have acquired the mec gene, which encodes PBP 2a, and the fem gene, which confer resistant to methicillin, penicillinaseresistant penicllins, and cephalosporins. ${ }^{12}$

The incidence of $S$. aureus bactermia (SAB), particularly bacteremia caused by MRSA strains, has increased dramatically in recent years in the United States and in some European countries. ${ }^{13,14}$ 
Determination of minimum inhibitory concentration (MIC) of antibiotics is an important aid to determine antibiotic resistant to bacteria. ${ }^{15}$ The MIC value of ceftriaxone was detected as $64 \mathrm{mg} / \mathrm{L}$ for 3 strains of $S$. aureus and $128 \mathrm{mg} / \mathrm{L}$ for 1 strain of $S$. aureus.

\section{Conclusion}

The most prevalent microorganisms in BSIs were gram positive bacteria such as $S$. aureus $69.5 \%$ and CoNS $26.9 \%$ among asymptomatic patients under course of hemodialysis. In the study, S. aureus was found to be resistant towards oxacillin and cephoxitin $(37.5 \%)$, and they are termed as MRSA. The finding of this study, however, gives an indication of which major pathogens causing BSI in patients and their drug susceptibilities. Further research could evaluate the impact of surveillance culture to prevent microorganisms' distribution in this population.

\section{Acknowledgments}

We would like to express my special thanks to National Kidney Center and the HD patients who were co-operative to give blood sample and their history. We are also thankful to National College for administrative support.

\section{Conflicts of interest}

Authors declare that there is no conflict of interest.

\section{References}

1. Fitzgibbons LN, Darcy LP, Kimberly M, et al. Management of Gram positive coccal Bacteremia and hemodialysis. Am J Kidney Dis. 2011;57(4):624-640.

2. Klevens RM, Morrison MA, Nadle J, et al. Invasive methicillinresistant Staphylococcus aureus infections in the United States. JAMA. 2007;298:1763-1771.

3. Forbes BA, Sahm DF, Weissfed AS. Bailey and Scott's Diagnostic Microbiology. $12^{\text {th }}$ ed. Mosby Inc: London, UK; 2007. 265-268, $277-$ 278, 285-312, 385-397, $780 \mathrm{p}$.
4. Fysaraki M, Samonis G, Valachis A, et al. Incidence, clinical, microbiological features and outcome of bloodstream infections in patients undergoing hemodialysis. Int J Med Sci. 2013;1(12):1632-1638.

5. Jaber BL. Bacterial infections in hemodialysis patients: pathogenesis and prevention. Kidney Int. 2005;67(6):2508-2519.

6. Taylor G, Gravel D, Johnston L. Prospective Surveillance for primary bloodstream infections occurring in Canadian hemodialysis units. Infect Control Hosp Epidemiol. 2002;23(12):716-720.

7. Chaudhry I, Chaudhry NA, Munir M, et al. Etiological Pattern of septicemia at Three Hospitals in Lahore. JCPSP. 2000;10:375-379.

8. Lee A, Mirrett S, Reller LB, et al. Detection of Bloodstream Infections in Adults: How Many Blood Cultures Are Needed? J Clin Microbiol. 2007;45(11):3546-3548.

9. Thiene G, Basso C. Pathology and pathogenesis of infective endocarditis in native heart valves. Cardiovasc Pathol. 2006;15(5):256-263.

10. Diekema DJ, Beekmann SE, Chapin KC, et al. Epidemiology and outcome of nosocomial and community-onset bloodstream infection. $J$ Clin Microbiol. 2003;41(8):3655-3660.

11. Manandhar S, Basnyat S, Sharma J, et al. Bacteriological profile of heart valves resected from infective endocarditis patients. $J$ Nepal Health Res Counc. 2009;7(2):108-111.

12. Jansen WT, Beitsman MM, Koeman CJ, et al. Novel mobile variants of staphylococcal cassette chromosome mec in Staphylococcus aureus. Antimicrobial Agents Chemother. 2006;50(6):2072-2078.

13. Shorr AF. Epidemiology of Staphylococcal resistnace. Clin Infec Dis. 2007;45(Suppl 3):S171-S176.

14. CLSI. Performance standards for antimicrobial susceptibility testing twenty-second informational supplement. CLSI Document M100-S23: Vol 32, Wayne, PA, USA; 2012.

15. Isalam MA, Alam MM, Choudhury ME, et al. Determination of minimum inhibitory concentration (MIC) of cloxacillin for selected isolates of Methicillin-Resistant Staphylococcus aureus (MRSA) with their Antibiogram. Bangl J Vet Med. 2008;6(1):121-126. 